
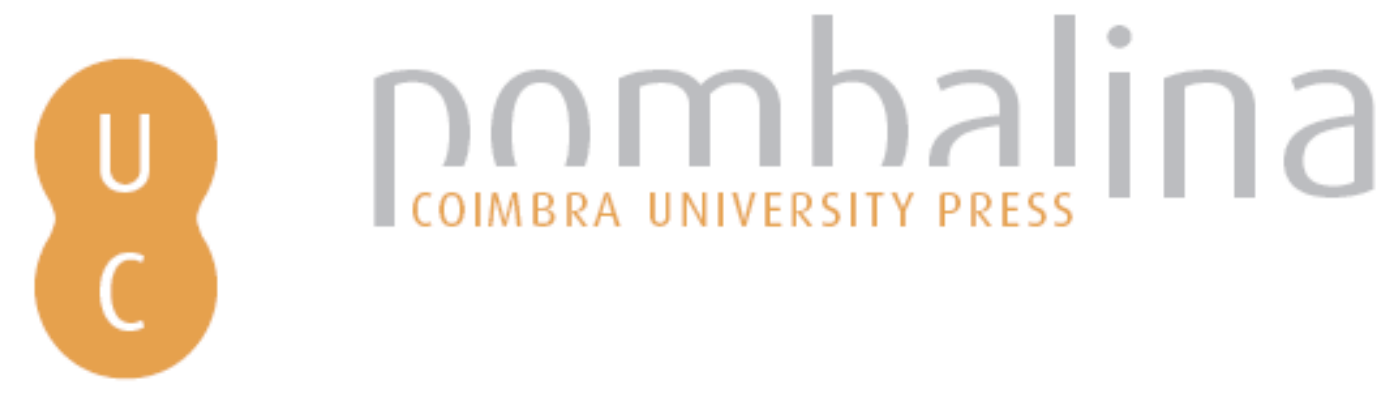

\title{
H. W. Kroto: curta biografia ou Sussex revisitada
}

Autor(es): $\quad$ Varandas, A. J. C.

Publicado por: Imprensa da Universidade de Coimbra; Gradiva

URL

persistente:

URI:http://hdl.handle.net/10316.2/32645

DOI:

DOI:http://dx.doi.org/10.14195/978-989-26-0389-6_2

Accessed : $\quad$ 26-Apr-2023 14:02:33

A navegação consulta e descarregamento dos títulos inseridos nas Bibliotecas Digitais UC Digitalis, UC Pombalina e UC Impactum, pressupõem a aceitação plena e sem reservas dos Termos e Condições de Uso destas Bibliotecas Digitais, disponíveis em https://digitalis.uc.pt/pt-pt/termos.

Conforme exposto nos referidos Termos e Condições de Uso, o descarregamento de títulos de acesso restrito requer uma licença válida de autorização devendo o utilizador aceder ao(s) documento(s) a partir de um endereço de IP da instituição detentora da supramencionada licença.

Ao utilizador é apenas permitido o descarregamento para uso pessoal, pelo que o emprego do(s) título(s) descarregado(s) para outro fim, designadamente comercial, carece de autorização do respetivo autor ou editor da obra.

Na medida em que todas as obras da UC Digitalis se encontram protegidas pelo Código do Direito de Autor e Direitos Conexos e demais legislação aplicável, toda a cópia, parcial ou total, deste documento, nos casos em que é legalmente admitida, deverá conter ou fazer-se acompanhar por este aviso. 
C I E N C I A I B E R T A

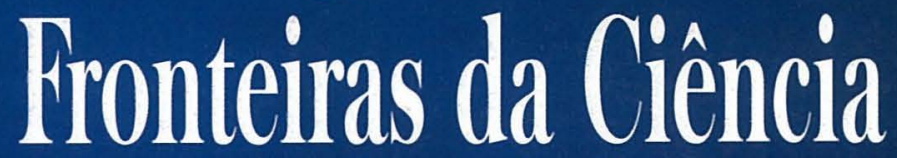

Desenvolvimentos Recentes - Desafios Futuros

RUI FAUSTO • CARLOS FIOLHAIS • JOÃO FILPE QUURRÓ

Coordenadores

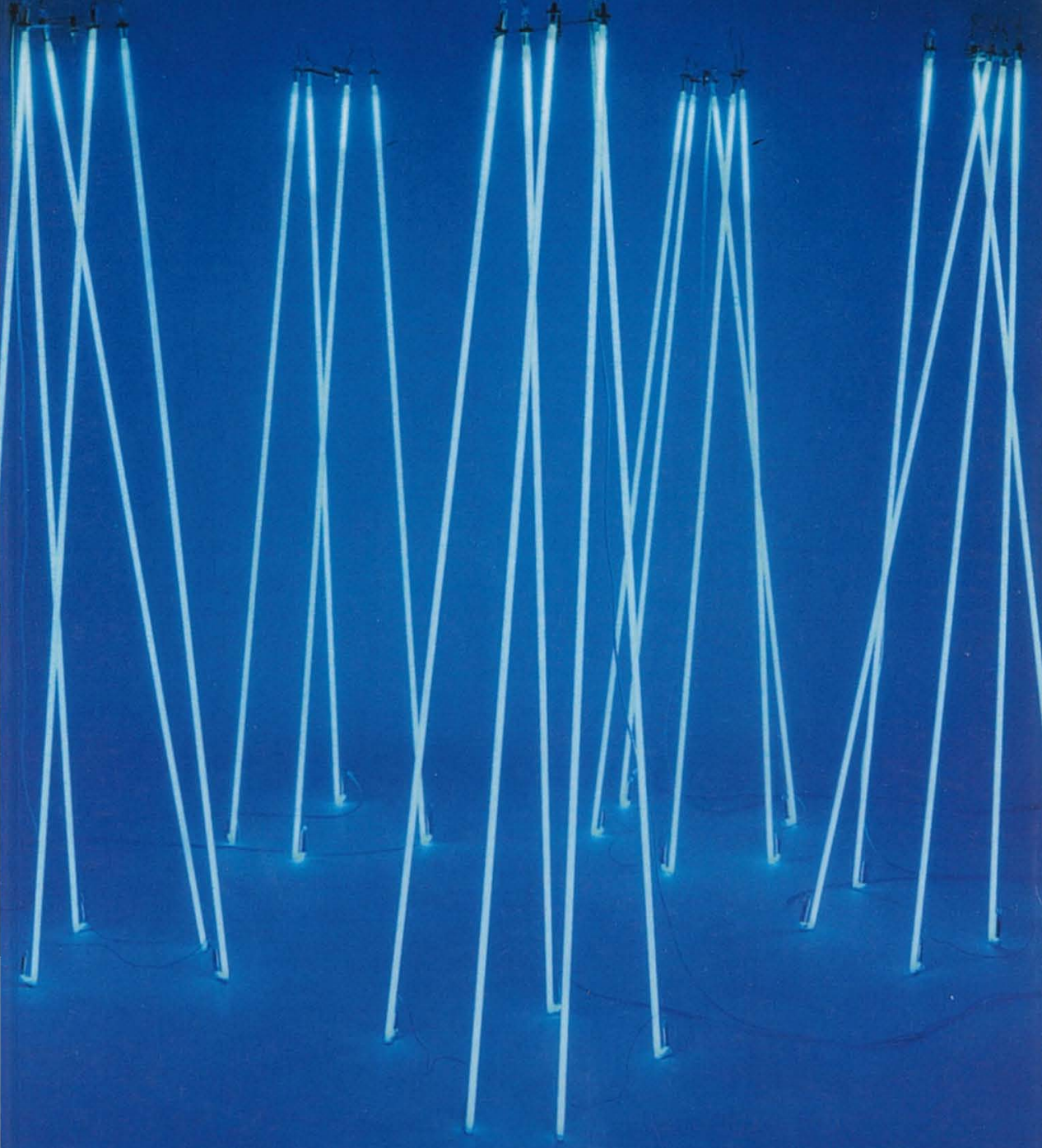

gradiva - Imprensa da Universidade de Coimbra 
(Página deixada propositadamente em branco) 


\section{RUI FAUSTO, CARLOS FIOLHAIS JOÃO FILIPE QUEIRÓ \\ Coordenadores}
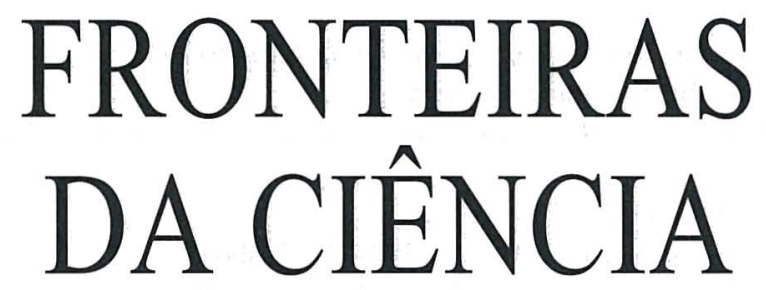

Desenvolvimentos Recentes Desafios Futuros 
(C) Gradiva - Publicações, L. da / Imprensa da Universidade de Coimbra, 2003 Coordenação editorial: Rui Fausto, Carlos Fiolhais e João Filipe Queiró Tradução: Jean Burrows, Vivien Burrows, Rui Fausto, Carlos Fiolhais e João Filipe Queiró

Revisão do texto: Isabel Pedrome

Capa: António Barros [Imprensa da Universidade. Coimbra], sobre imagem de «Águas Vivas», escultura de Silvestre Pestana, 2001

Foto: António Alves; Infografia: ESTÍMULUS [design]; Cortesia: Galeria Alvarez-Arte Contemporânea

Paginação: António Resende e Paula Isabel Jorge

Impressão e acabamento: G.C. - Gráfica de Coimbra, L. ${ }^{d a}$

Reservados os direitos para Portugal por:

Gradiva - Publicações, L. ${ }^{\text {da }}$ e Imprensa da Universidade de Coimbra

Gradiva - Publicações, L.da

Rua Almeida e Sousa, 21, r/c, esq.•1399-041 Lisboa

Telefs. $213974067 / 8 \cdot 213971357 \cdot 213953470$

Fax $213953471 \cdot$ Email: gradiva@ip.pt

URL: http://www.gradiva.pt

Imprensa da Universidade de Coimbra

Rua Antero de Quental, 195 • 3000-033 Coimbra

Telefs. 351239853110

Fax 3512398531 19・e-mail: fjrpress@ci.uc.pt

URL: http://www.imp.uc.pt

ISBN: 972-662-923-3

1." edição: Agosto de 2003

Depósito legal n. ${ }^{\circ} 199$ 463/2003 
A. J. C. Varandas

Departamento de Química

Faculdade de Ciências e Tecnologia

Universidade de Coimbra

\section{H. W. Kroto: curta biografia ou Sussex revisitada}

Quando recebi o convite para integrar o painel de debate sobre a palestra do Professor Sir Harold W. Kroto senti-me dividido nas minhas emoções. Por um lado, apreensivo, pois era a primeira vez que me era pedido que falasse sobre uma molécula com mais do que uns poucos átomos, o que me levou a pensar que até poderia não alcançar a verdadeira perspectiva da química do fulereno. Por outro lado, sentia-me honrado e encantado por poder falar sobre o trabalho de um distinto cientista e Prémio Nobel.

Encontrei Harry Kroto pela primeira vez no Outono de 1973, quando cheguei à Universidade de Sussex para aí fazer o meu doutoramento, sob a supervisão do Professor John N. Murrell, o que aconteceu seis anos depois da chegada de Harry Kroto a Sussex, onde iniciava a sua carreira académica. Antes disso, em 1961, o Harry Kroto tinha-se licenciado em Química na Universidade de Sheffield, e em 1964 concluíra o seu doutoramento sobre espectros electrónicos de alta resolução de radicais livres produzidos por fotólise de relâmpago, pesquisa realizada sob a supervisão do Professor Richard N. Dixon. Tinha também realizado dois anos de investigação de pós-doutoramento em espectroscopia electrónica e de microondas no National Research Council (1964-1966), no Canadá, e nos Bell Telephone Laboratories (1966-1967), onde se dedicou ao estudo das interacções em fase líquida por espectroscopia de Raman e levou a 
cabo estudos de Química Quântica. Harry Kroto, na altura com 34 anos e professor em Sussex, cedo despertou a minha atenção durante os seminários de Química Física que eram organizados semanalmente na School of Chemistry and Molecular Sciences*. O seu talento tornou-se para mim particularmente visível no ano seguinte, quando deu uma série de palestras sobre espectroscopia rotacional. Como recém-chegado de Coimbra, com uma licenciatura em Engenharia Química e mal tendo começado a minha pesquisa sobre a teoria das forças intermoleculares, senti-me muitas vezes pouco à vontade para seguir os detalhes do tratamento algébrico do momento angular que Harry ensinava entusiasticamente. Felizmente, deu a alguns de nós umas provas não corrigidas do livro Molecular Rotation Spectra, que tinha acabado de escrever. Estas revelaram-se essenciais para o meu trabalho e, claro está, muito do que então aprendi acabou por ter um papel importante na investigação científica que tenho vindo a realizar. Estou agradecido a Harry Kroto por ter, sem dúvida nenhuma, contribuído para a minha aprendizagem. E agora uma pequena confissão: as provas não corrigidas n. ${ }^{\circ} 5$ de Molecular Rotation Spectra ${ }^{1}$ nunca foram devolvidas; na verdade, ainda as guardo na minha estante!

Durante os meus anos em Sussex (1973-1976) a pesquisa de Kroto centrava-se na criação e caracterização espectroscópica de novas moléculas, em particular de espécies instáveis que continham ligações múltiplas lábeis. $\mathrm{O}$ seu trabalho levou à produção das primeiras moléculas com ligações duplas de carbono e fósforo, assim como ao desenvolvimento dos primeiros compostos com ligações triplas entre estes elementos. Poderia adivinhar-se a onda de progresso na pesquisa de Kroto pela sua alegria nos intervalos do chá na School of Chemistry and Molecular Sciences. Harry parecia muitas vezes excitado, emocionado, e na maior parte das vezes tinha um sorriso no rosto: tal contrastava com a maior parte de nós (estudantes), que não conseguíamos ver o trabalho de um modo tão promissor.

Uma outra lembrança dos meus anos em Sussex, que recordo muitas vezes aos meus alunos, diz respeito ao elevado número de teses de licenciatura expostas numa sala perto da biblioteca de química. Era com satisfação que me sentava nessa sala e as folheava. Podia ter uma ideia do trabalho feito por várias gerações de estudantes e avaliar a produtividade da ainda jovem Universidade de Sussex. Com efeito, um

* Actualmente School of Chemistry, Physics and Environmental Science. 
tema enfaticamente referido por Kroto durante a sua lição por ocasião da atribuição do Prémio Nobel $^{2}$ diz respeito ao chamado Chemistry by Thesis Course em Sussex. Diz ele: Uma licenciatura em Ciências poderia ser obtida tendo por base uma tese que um estudante não licenciado escrevesse sobre um tópico de investigação levada a cabo sob a supervisão de dois tutores de diferentes disciplinas da área da química (por exemplo, síntese química e espectroscopia) ${ }^{3}$. Continuando a citação, o curso reconheceu que a avaliação tradicional das capacidades de um estudante - e, particularmente, das suas capacidades futuras - era um indicador artificial e muitas vezes insatisfatório ${ }^{4}$. Não posso estar mais de acordo. De facto, foi durante um destes cursos que Kroto e um dos seus alunos prepararam o $\mathrm{HC}_{5} \mathrm{~N}$, considerado um percursor das suas notáveis descobertas posteriores sobre a química do fulereno ${ }^{5}$.

Com a descoberta do $\mathrm{HC}_{5} \mathrm{~N}$, surgiu também o interesse de Kroto pelas estrelas gigantes negras e gigantes vermelhas (química interestelar). De facto, as vastas nuvens negras que se encontram entre as estrelas atraíram não só o seu interesse, mas também o de muitos outros cientistas, visto abrigarem uma variedade de moléculas (metanol, monóxido de carbono, formaldeído, etanol, cianeto de hidrogénio, ácido fórmico, formamida, etc.) que, em conjunto, poderiam ser consideradas um contentor primordial da sopa prebiótica ${ }^{6}$. Apesar de existirem indicações de que um átomo adicional de carbono reduziria a abundância da molécula por um factor de aproximadamente dez (isto é, o $\mathrm{HC}_{5} \mathrm{~N}$ teria um factor de abundância 100 vezes menor que o cianoetino, $\mathrm{HC}_{3} \mathrm{~N}$, já detectado ${ }^{7}$ ) a tenacidade da visão de Kroto, que sustentava que nenhuma regra devia ser suposta como certa antes de ser provada experimentalmente, levou à surpreendente descoberta, através da radioastronomia, de cianopolininos $\left(\mathrm{HC}_{n c} \mathrm{~N}\right)$ com longas $\left(n_{\mathrm{c}} \leq 11\right)$ cadeias de átomos de carbono no espaço interestelar ${ }^{8}$.

No entanto, foi em Setembro de 1985, durante uma visita de dez dias ao laboratório do Professor Rick Smalley, na Universidade de Houston, que a criação e o isolamento de grandes agregados de carbono sem ligações pendentes ocorreram pela primeira vez. Juntamente com o Professor Robert Curl, da Universidade de Rice, o Professor Rick Smalley e os seus estudantes J. R. Heath e S. C. O' Brien, Kroto descobriu ${ }^{9}$ que o $\mathrm{C}_{60}$ podia ser produzido de uma forma inigualavelmente estável, partindo apenas da vaporização da grafite por laser num jacto pulsionado de hélio. Durante as suas experiências, fez a intrigante descoberta de que, sob condições particulares de agregação, o pico a 720 era de longe o mais intenso no espectro de massa. Relembrando a sua visita à Expo $67 \mathrm{em}$ Monreal (32 anos antes da Expo 98, em Lisboa), Kroto lembrou-se então das cúpulas de Buckminster Fuller ${ }^{10}$, e propôs dar ao seu agregado de 
carbono um nome baseado nas invenções de Fuller, isto é, buckminsterfulereno ou fulereno-60. Apesar de se pensar na altura que a estabilidade do $\mathrm{C}_{60}$ se pudesse dever ao carácter singular das propriedades geodésicas e electrónicas inerentes à sua estrutura em forma de gaiola icoseidal truncada, existe hoje uma riqueza de informação experimental e teórica bastante convincente que apoia independentemente a singular estabilidade físico-química ${ }^{11}$ da molécula do buckminsterfulereno. De facto, a existência dos fulerenos como família de compostos já está igualmente estabelecida e a designação fulereno- $n_{\mathrm{c}}$ é correntemente usada para um fulereno com um número $n_{\mathrm{c}}$ de átomos de carbono.

Durante os anos que se seguiram à descoberta ${ }^{12}$ do buckminsterfulereno, tinha por hábito visitar de vez em quando a Universidade de Sussex, uma vez que John Murrell e alguns dos seus colaboradores, incluindo eu próprio, estávamos a acabar de escrever um livro ${ }^{13}$ intitulado Molecular Potential Energy Functions. Nessa altura podia ver o movimento no laboratório de Harry Kroto e lembro-me de uma ocasião em que ele me levou a ver as suas cúpulas de Buckminster Fuller, ou, por outras palavras, os seus modelos moleculares de fulerenos, dos menores aos maiores ${ }^{14}$. O zoo completo de fulerenos é agora facilmente acessível, bastando extrair as coordenadas cartesianas dos átomos da Internet ${ }^{15}$. Uma vez colocados no espaço a três dimensões, ficam mesmo muito bonitos, apesar de devermos ter em conta que os fulerenos não são só bonitos, conforme Kroto costuma salientar. No entanto, o buckminsterfulereno, $\mathrm{C}_{60}$, é um fulereno único: a sua gaiola apresenta simetria máxima (todos os átomos ocupam posições equivalentes nas extremidades de um icosaedro truncado) e a forma de uma bola de futebol. Por isso é vulgarmente conhecido por buckyball.

É evidente que a descoberta do buckminsterfulereno não podia deixar a comunidade científica indiferente. No Outono de 1990, um novo tipo de fase condensada do carbono baseada no $\mathrm{C}_{60}$ foi sintetizada pela primeira vez por Krätschmer, Lamb, Fostiropoulos e Huffman ${ }^{16}$. Tal como aconteceu com Kroto, as suas motivações iniciais provinham dos seus interesses em astrofísica. O maior sucesso de Krätschmer et al. ${ }^{17}$ foi terem descoberto um método para preparar grandes quantidades de $\mathrm{C}_{60}$, que até então estava apenas disponível em pequenas quantidades na fase gasosa ${ }^{18}$. Apesar de parecer uma ocorrência natural, tal descoberta encerra algo de trágico. Com efeito, Kroto tinha estado a investigar um método mais simples para a produção e isolamento do $\mathrm{C}_{60}$. Ironicamente, foi até convidado para avaliar o importante trabalho de Krätschmer et al. ${ }^{19} \mathrm{Nas}$ suas próprias palavras: Pensei suicidar-me, mas preferi ir almoçar ${ }^{20}$. O seu carácter e o seu pacato ar britânico continuaram a acompanhá-lo em 
muitas descobertas importantes, ao mesmo tempo que lhe permitiram reconhecer o mérito alheio: Krätschmer, Lamb, Fostiropoulos e Huffman extraíram realmente $C_{60}$, e em Sussex ficámos muito próximos de alcançar tal objectivo quase em simultâneo ${ }^{21}$.

Em resumo, os fulerenos podem agora ser objecto de pesquisas intensas por diversos grupos no mundo inteiro. De facto, foram identificados em amostras geológicas ${ }^{22}$, e estão provavelmente distribuídos em grandes quantidades no universo, particularmente no fluxo de partículas expelidas pelas estrelas de carbono ${ }^{23}$. Devido à sua notável estabilidade ${ }^{24}$, os fulerenos atraem naturalmente muito interesse para aplicações práticas $^{25}$. Por exemplo, a descoberta por Hebard e colaboradores ${ }^{26}$ de que o intercalamento de metais alcalinos em $\mathrm{C}_{60}$ com estequiometria $\mathrm{M}_{3} \mathrm{C}_{60}$ (onde $\mathrm{M}=\mathrm{K}, \mathrm{Rb}, \mathrm{Cs}$ ) modifica substancialmente as propriedades electrónicas do fulereno hospedeiro, produzindo supercondutores a temperaturas relativamente altas $\left(18 \mathrm{~K} \leq \mathrm{T}_{\mathrm{C}} \leq 33 \mathrm{~K}\right)$, veio estimular a pesquisa sobre materiais relacionados com o buckminsterfulereno.

Como teórico não posso deixar de mencionar os muitos estudos teóricos citados na literatura sobre fulerenos ${ }^{27}$. De facto, como acontece na maioria das descobertas, existe uma pré-história do estudo de gaiolas moleculares, de que fazem parte algumas previsões teóricas ${ }^{28}$. Citando Kroto, foram precisos mais ou menos 15 anos até as conjecturas imaginativas de Osawa e Yoshida ${ }^{29}$ e de Bochvar e Gal'pern ${ }^{30}$ serem concretizadas com a observação do sinal do espectro de massa do $C_{60}$ em $1985^{31}$. Não é portanto surpreendente, pelo menos para mim, que já a tinha antecipado em Janeiro de 1992 durante um jantar com Kroto em Lisboa, que a sua descoberta histórica viesse a culminar num Prémio Nobel: Kroto partilhou-o com Smalley e Curl em 1996.

Kroto recebeu muitos prémios e distinções honoríficas. Foi Tildon Lecturer (Royal Society of Chemistry) (1981-1982) e recebeu os seguintes prémios e medalhas: Fellow of the Royal Society (1990), Royal Society Research Fellowship (1991), International Prize for New Materials (American Physical Society) (1992), Italgas Prize for Innovation in Chemistry (1992), Longstaff Medal (Royal Society of Chemistry) (1993), Hewllet-Packard Europhysics Prize (1994); prémio Moet Henessy/Louis Vuitton Science Pour 1'Art (1994). Foi também nomeado Membro da Academia Europeia e Doutor Honoris Causa nas Universidades de Bruxelas (1992), de Estocolmo (1992) e de Limburgo (1993). Semelhantes cargos honorários foram-lhe dados pela Universidade de Sheffield (1995) e pela Universidade de Kingston (1995). Kroto é além disso presidente do Editoral Board da Chemical Society Reviews. 
Em conclusão, para além do seu interesse científico e tecnológico, a simetria dos fulerenos confere-lhes um carisma especial. O fascínio dos homens pela simetria remonta aos sólidos platónicos e continua com o buckminsterfulereno. Devido à sua estrutura elegantemente simples e bastante simétrica, trata-se de uma das moléculas mais populares e talvez mais estudadas da química. Para além disso, a cavidade vazia dentro das gaiolas moleculares do fulereno levou à criação da química endoédrica, uma área actualmente em grande desenvolvimento ${ }^{32}$. Como Kroto expressou em 1991, a química do fulereno é uma área de estudo apaixonante e a expectativa de novos materiais com novas propriedades torna-a ainda mais promissora. Com certeza, um novo ciclo do carbono, pós-buckminsterfulereno, parece ter sido encontrado no mundo da química da noite para o dia ${ }^{33}$.

\section{Referências}

${ }^{1}$ Kroto, H. W., Molecular Rotation Spectra, Wiley, Londres, 1974.

${ }^{2}$ Kroto, H. W., Angew. Chem. Ind. Ed. Engl., 1997, 36, 1578.

3 Idem, ibidem.

${ }^{4}$ Idem, ibidem.

5 Idem, ibidem.

${ }^{6}$ Kroto, H. W., Int. Rev. Phys. Chem., 1981, 1, 309.

7 Turner, B. E., Astrophys. J., 1971, 163, L35.

${ }^{8}$ Kroto, H. W.; Allaf, A. W.; Balm, S. P., Chem. Rev., 1991, 91, 1213.

9 Kroto, H. W.; Heath, J. R.; O’Brien, S. C.; Curl, R. F.; Smalley, R. E., Nature, 1985, 318, 162.

${ }^{10}$ Fuller, R. B., Inventions - The Patented Works of Buckminster Fuller; St. Martin's Press: New York, 1983.

${ }^{11}$ Kroto, H. W., Angew. Chem. Ind. Ed. Engl., 1997, 36, 1578. / Kroto, H. W., Allaf, A. W.; Balm, S. P., Chem. Rev., 1991, 91, 1213. / Dresselhaus, M. S.; Dresselhaus, G.; Ecklund, P. C., Science of Fullerenes and Carbon Nanotubes. Academic Press: San Diego, 1996.

${ }^{12}$ Kroto, H. W.; Heath, J. R.; O’Brien, S. C.; Curl, R. F.; Smalley, R. E., Nature, 1985, 318, 162.

${ }^{13}$ Murrell, J. N.; Carter, S.; Farantos, S. C.; Huxley, P.; Varandas, A. J. C., Molecular Potential Energy Functions; Wiley: Chichester, 1984.

${ }^{14}$ Kroto, H. W., Chemistry in Britain, 1990, 26, 40.

${ }^{15}$ As coordenadas cartesianas de muitas moléculas de fulerenos podem ser obtidas em: ftp://informeister.osc.edu/pub/chemistry/data/fullerenes.

${ }^{16}$ Krätschmer, W.; Lamb, L. D.; Fostiropoulos, K.; Huffman, D. R., Nature, 1990, 347, 354. 
17 Idem, ibidem.

18 Kroto, H. W.; Heath, J. R.; O’Brien, S. C.; Curl, R. F.; Smalley, R. E., Nature, 1985, 318, 162. / Curl, R. F.; Smalley, R. E., Science, 1988, 242, 1017. 19 Krätschmer, W.; Lamb, L. D.; Fostiropoulos, K.; Huffman, D. R., Nature, $1990,347,354$.

${ }^{20}$ Kroto, H. W., Angew. Chem. Ind. Ed. Engl., 1997, 36, 1578.

21 Idem, ibidem

${ }^{22}$ Kroto, H. W.; Heath, J. R.; O’Brien, S. C.; Curl, R. F.; Smalley, R. E., Nature, 1985, 318, 162.

${ }^{23}$ Kroto, H. W.; Heath, J. R.; O’Brien, S. C.; Curl, R. F.; Smalley, R. E., Nature, 1985, 318, 162.

24 Kroto, H. W., Nature, 1987, 329, 529.

25 Kroto, H. W., Angew. Chem. Ind. Ed. Engl., 1997, 36, 1578. / Kroto, H. W.; Allaf, A. W.; Balm, S. P., Chem. Rev., 1991, 91, 1213. / Dresselhaus, M. S.; Dresselhaus, G.; Ecklund, P. C., Science of Fullerenes and Carbon Nanotubes. Academic Press: San Diego, 1996.

${ }^{26}$ Haddon, R. C.; Hebard, A. F.; Rosseinsky, M. J.; Murphy, D. W.; Duclos, S. J.; Lyons, K. B.; Miller, B.; Rosamilia, J. M.; Fleming, R. M.; Kortan, A.; Glarum, S.; Makhija, A.; Muller, A.; Eick, R.; Zahurak, S.; Tycko, R.; Dabbagh, G.; Thiel, F., Nature, 1991, 350, 320. / Hebard, A. F., Phys. Today, 1992, 45, 26.

27 Kroto, H. W., Angew. Chem. Ind. Ed. Engl., 1997, 36, 1578. / Kroto, H. W.; Allaf, A. W.; Balm, S. P., Chem. Rev., 1991, 91, 1213. / Dresselhaus, M. S.; Dresselhaus, G.; Ecklund, P. C., Science of Fullerenes and Carbon Nanotubes. Academic Press: San Diego, 1996.

28 Jones, D. E. H., New Sci., 1966, 32, 245. / Osawa, E., Kagaku (Kyoto), 1970, 25,854 .

29 Osawa, E., Kagaku (Kyoto), 1970, 25, 854.

30 Bochvar, D. A.; Gal'pern, E. G., Dokl. Akad. Nauk SSSR, 1973, 209, 610. I /Stankevich, I. V.; Nikerov, M.V.; Bochvar, D.A., Russ. Chem. Rev., 1984, 53, 640 .

31 Kroto, H. W.; Allaf, A. W.; Balm, S. P., Chem. Rev., 1991, 91, 1213.

32 Kroto, H. W., Angew. Chem. Ind. Ed. Engl., 1997, 36, 1578. / Kroto, H. W.; Allaf, A. W.; Balm, S. P., Chem. Rev., 1991, 91, 1213. / Dresselhaus, M. S.; Dresselhaus, G.; Ecklund, P. C., Science of Fullerenes and Carbon Nanotubes. Academic Press: San Diego, 1996.

${ }^{33}$ Kroto, H. W.; Allaf, A. W.; Balm, S. P., Chem. Rev., 1991, 91, 1213. 
 \\ A palavra "fronteiras" pode ser tomada em} diferentes sentidos. Pode referir-se aos limites, necessariamente provisórios, entre o conhecido e o desconhecido, ou aos limites entre o possivel e o impossivel, e, dentro do possivel, entre o desejável e 0 indesejável. Fronteiras podem também ser as delimitações, nem sempre nítidas, entre ciência e não-ciência, e dentro da ciência, entre as várias disciplinas. Quais são então as fronteiras da ciência?

Neste livro, a resposta a esta pergunta é dada, segundo as mais diferentes perspectivas, por um conjunto notável de personalidades, cientistas ou não, entre as quais se contam três Prémios Nobel.

Rui Fausto, Carlos fiolhais e JoÃo Fillipe Queiró são, respectivamente, professores de Química, Física e Matemática na Faculdade de Ciências e Tecnologia da Universidade de Coimbra. 